Artigo Original

\title{
Composição corporal, maturação sexual e desempenho motor de jovens praticantes de handebol
}

\author{
Rodolfo André Dellagrana ${ }^{1}$ \\ Michael Pereira da Silva ${ }^{2}$ \\ André de Camargo Smolarek ${ }^{1}$ \\ Rodrigo Bozza ${ }^{1}$ \\ Antonio Stabelini Neto ${ }^{1}$ \\ Wagner de Campos ${ }^{3}$ \\ ${ }^{1}$ Centro de Pesquisa em Exercício e Esporte; Programa de Pós-Graduação em \\ Educação Física da Universidade Federal do Paraná, Curitiba, PR, Brasil \\ ${ }^{2}$ Centro de Pesquisa em Exercício e Esporte. Universidade Federal do Paraná, Curitiba, \\ PR, Brasil \\ ${ }^{3}$ Centro de Pesquisa em Exercício e Esporte; Departamento de Educação Física da \\ Universidade Federal do Paraná, Curitiba, PR, Brasil
}

Resumo: O presente estudo teve como objetivo investigar a associação entre composição corporal e maturação sexual com o desempenho motor de jovens praticantes de handebol de ambos os sexos. Foram avaliados 47 adolescentes com idades entre 12 e 17 anos. A avaliação da maturação sexual foi realizada através do teste de auto-avaliação. As variáveis antropométricas de massa corporal e estatura foram mensuradas, além das dobras cutâneas do tríceps e da panturrilha, para a estimativa do percentual de gordura (\%G). Para a análise do desempenho motor foi realizada uma bateria de testes motores: impulsão horizontal $(\mathrm{IH})$, flexão de braços, abdominal, sentar e alcançar e corrida de vai e vem - shuttle run (agilidade). Observou-se uma relação inversa entre \%G e IH (r=-0,42), e a agilidade (Ag) obteve relação positiva com o \%G ( $r=0,61)$. A correlação entre maturação e IH foi positiva $(r=0,32)$, e com a Ag negativa $(r=-0,27)$. O sexo explicou 32,8\% da variabilidade da IH e em conjunto com o \%G e maturação o valor foi de $36,5 \%$. Para a Ag a variabilidade foi explicada em 39,4\% pelo sexo, juntamente com o \%G e a maturação este valor foi elevado à $55 \%$. Apesar da contribuição preditora do sexo em conjunto com o \%G e a maturação para as variáveis de $\mathrm{IH}$ e Ag, os valores foram baixos, podendo não atribuir as alterações destas variáveis motoras apenas às variáveis independentes analisadas.

Palavras-chave: Handebol. Composição corporal. Maturação sexual. Desempenho motor.

\section{Body composition, sexual maturation and motor performance the young practitioners handball}

\begin{abstract}
This study aimed to investigate the association between body composition and sexual maturity with the motor performance of male and female youngsters playing handball. 47 adolescents between 12 and 17 years old were evaluated. The assessment of sexual maturation was performed using selfassessment. The anthropometric variables of body mass and height were measured, and skinfolds of triceps and calf, to estimate the percentage of body fat (\% BF). For the analysis of motor performance was performed a battery of motor tests: horizontal jump (HJ), arm flexion, abdominal, sit and reach and shuttle run (agility). There was an inverse relationship between \%BF and $\mathrm{IH}(\mathrm{r}=-0,42)$, and agility (Ag) obtained positive relationship with \%BF $(r=0,61)$. The correlation between maturation and $\mathrm{IH}(0,32)$ was positive, and the Ag negative $(-0,27)$. Sex explained $32.8 \%$ of the variability of $\mathrm{IH}$ and in conjunction with the \%BF and maturation the value was $36.5 \%$. For $\mathrm{Ag}$ the variability was explained in $37.6 \%$ by the sex, along with the $\% \mathrm{BF}$ and maturation of this value was raised to $55 \%$. Despite the predictive contribution of the sex together with the \%BF and maturation of the variables of $\mathrm{IH}$ and $\mathrm{Ag}$, the values were low. It can not be attributed the variation of the motor variables only to independent variables.
\end{abstract}

Key Words: Handball. Body composition. Sexual maturation. Motor performance.

\section{Introdução}

A adolescência é o período onde ocorrem as condições mais favoráveis para o desenvolvimento de todas as capacidades físicas, desde que ocorra uma ação racional, pedagógica e sistemática (ALTINI NETO et al., 2006). Vários aspectos de um indivíduo sofrem influências da maturação biológica como a composição corporal, o crescimento e o desempenho motor (MARTIN et al., 2001). Nesta fase, a idade cronológica não 
é um parâmetro seguro para a caracterização biopsicossocial do indivíduo, pois indivíduos da mesma idade podem apresentar fases maturacionais diferentes.

Variações no tamanho corporal e no desempenho motor relacionados às diferenças inter-individuais da maturação biológica são de grande valor dentro da prática esportiva para a população jovem (MALINA et al., 2007), visto que, foi observado que o nível de desempenho motor apresenta correlação positiva com o estágio de maturação biológica (FERREIRA et al., 1990). E ainda, outras evidências científicas demonstram que dentro da prática esportiva, jovens em estágios maturacionais avançados em relação a indivíduos de um mesmo grupo de treinamento ou categoria com maturação atrasada, apresentam vantagens no desempenho (MALINA et al., 2000; HELSEN et al., 2000).

Além disso, importantes mudanças fisiológicas ocorrem durante a infância e a adolescência, sendo que este processo acontece com antecedência no sexo feminino comparado ao sexo masculino (MACHADO; BARBANTI, 2007). Desta forma, diferenças são encontradas entre os sexos, nas variáveis antropométricas, no desempenho motor e na composição corporal onde meninas apresentam maior quantidade de gordura e menor massa magra por volta dos sete aos 15 anos de idade (SILVA, 2002; GUEDES; GUEDES, 1993).

Seguindo este contexto, na maioria das modalidades esportivas a mensuração da composição corporal é de fundamental importância, pois o grande interesse da avaliação da composição do corpo no exercício, empregando a diferenciação entre massa gorda e massa livre de gordura, se dá pelo seu efeito significante no desempenho atlético e nas capacidades biomecânicas e fisiológicas (KRAEMER et al., 1999).

Neste sentido, a adiposidade corporal está inversamente relacionada ao desempenho motor, em crianças de ambos os sexos, principalmente nas variáveis de flexão e extensão de braços e salto em distância parado (FERREIRA; BÖHME, 1998). Verificam-se ainda correlações negativas da massa corporal com o desempenho em atividades de saltos e corridas, mostrando que o controle da composição corporal pode ser considerado como um fator importante para obter melhores índices no desempenho motor de crianças e adolescentes (MALINA; BOUCHARD, 1991).

Considerando estes fatores e os poucos estudos encontrados na literatura com jovens praticantes da modalidade de handebol o presente estudo teve como objetivos: a) analisar as diferenças das variáveis antropométricas e do desempenho motor entre os sexos; b) verificar as diferenças das variáveis antropométricas, composição corporal e desempenho motor entre indivíduos púberes e pós púberes; c) investigar a associação entre sexo, composição corporal e maturação sexual com o desempenho motor de jovens praticantes de handebol.

\section{Amostra}

\section{Métodos}

A amostra do estudo foi composta por 47 adolescentes (17 meninos e 30 meninas) com idades entre 12 e 17 anos, matriculados em uma instituição de ensino particular do município de Curitiba, Paraná. Todos participavam de treinamento sistematizado de handebol, oferecido no período "contra turno" das aulas, com freqüência de três vezes semanais e duração de 90 minutos/dia.

Anteriormente ao início das avaliações foi entregue aos pais ou responsáveis dos participantes um termo de consentimento livre e esclarecido (TCLE), com a finalidade de conhecer o protocolo utilizado no decorrer da pesquisa. Este estudo foi aprovado pelo Comitê de Ética da Universidade Federal do Paraná (protocolo 3185.0.000.091-08).

\section{Instrumentos e procedimentos}

Como indicador da maturação biológica, foi utilizada a avaliação dos estágios de maturação sexual propostos por Tanner (1962) compostos por cinco estágios, classificados da seguinte forma: estágio I - pré-púbere, estágios II, III e IV púberes, e estágio $\mathrm{V}$ - pós-púberes. $\mathrm{O}$ teste foi aplicado na forma de auto-avaliação por figuras do desenvolvimento da pilosidade pubiana, dado que esse é considerado um indicador confiável da maturação biológica (MARTIN et al., 2001).

As variáveis antropométricas de massa corporal (MC) e estatura foram mensuradas com uma balança antropométrica da marca Plenna® e uma fita métrica com escala de medida de $0,1 \mathrm{~cm}$ 
fixada à parede sem rodapé e perpendicular ao solo, respectivamente, seguindo a procedimentos propostos por Gordon et al. (1991). A partir destas medidas foi realizado o cálculo do Índice de Massa Corporal (IMC), através da razão entre a massa corporal e a estatura ao quadrado.

Para a estimativa do percentual de gordura $(\% \mathrm{G})$ foram mensuradas as dobras cutâneas do tríceps e da panturrilha, sendo realizadas sempre no hemicorpo direito. A equação de Slaughter et al. (1988) foi utilizada, pois leva em consideração a idade e o estágio maturacional.

$\mathrm{Na}$ análise do desempenho motor foi realizada uma bateria de testes motores (GUEDES; GUEDES, 2006), com indicadores de força explosiva de membros inferiores (impulsão horizontal), força/resistência muscular (flexão de braços e abdominal modificado), flexibilidade (sentar e alcançar) e agilidade (corrida de vai e vem - shuttle run):

Impulsão horizontal - os indivíduos permaneceram de pé, na marca zero de uma fita métrica fixada ao solo, com os pés paralelos em pequeno afastamento lateral. Sem realizar corrida de aproximação, podendo fazer apenas balanceio dos braços, procuraram saltar o mais distante possível. A distância entre a marca zero até a ponta do pé localizado mais próximo do ponto inicial foi registrada.

Flexão de braços - os indivíduos permaneceram em decúbito ventral, para o sexo feminino sugere-se que as pernas fiquem semiflexionadas com os joelhos apoiados ao chão, e para o sexo masculino é sugerido que as pernas fiquem estendidas. Assim são realizadas repetições máximas de flexão de braços.

Abdominal modificado 1 minuto - os indivíduos ficaram em decúbito dorsal, pernas flexionadas, joelhos formando um ângulo de $90^{\circ}$, pés fixados pelo avaliador e mãos cruzadas sobre o tórax. Ao sinal do avaliador, foram realizados movimentos de antero-flexão de tronco, encostando os cotovelos nas coxas, e voltando à posição inicial, com as escápulas encostando no solo. $O$ número máximo de repetições corretas, realizadas em 1 minuto foi registrado.

Sentar e alcançar - o avaliado ficou sentado, com a planta dos pés encostando-se a uma caixa de madeira (banco de Wells), com as costas, e com os membros inferiores em extensão total. Em seguida, ele foi instruído a realizar flexão máxima do tronco, para atingir com as mãos, sem flexionar as pernas, o ponto mais distante possível.

Corrida de vai e vem (shuttle run) - Em um espaço de 9,14 metros com dois blocos de madeira colocados atrás da linha contrária do inicio do teste. $O$ avaliado inicia em posição de pé, atrás da linha de partida. Ao comando do avaliador, corre em direção aos blocos, pega um, retorna a linha de partida colocando o bloco atrás desta linha, repetindo esta movimentação com o outro bloco. Foi registrado o tempo gasto para pegar os blocos e levá-los a linha de partida.

\section{Análise estatística}

Utilizou-se inicialmente a estatística descritiva para a caracterização da amostra (médias e desvios padrão, freqüência relativa e absoluta). $O$ teste de Shapiro-Wilk foi utilizado para verificação da normalidade das variáveis analisadas. Após a constatação da normalidade das variáveis o teste t para amostras independentes foi utilizado para comparação de médias entre os gêneros e entre os indivíduos púberes e pós-púberes.

Levando em consideração a amostra total foram calculados os coeficientes de correlação de Pearson para a relação do $\% \mathrm{G}$ com as varáveis motoras e a correlação de Spearman para a relação dos estágios maturacionais com as variáveis de desempenho motor. $\mathrm{Na}$ seqüência foram realizadas análises de regressão linear "stepwise", para analisar as inter-relações e a predição das variáveis independentes sobre os componentes do desempenho motor.

Todos os dados foram analisados no pacote estatístico SPSS versão 13.0 para Windows, adotando um nível de significância de 0,05.

\section{Resultados}

Na tabela 1, estão descritos os valores médios das variáveis antropométricas e do desempenho motor e as diferenças entre os sexos. Meninos apresentam maior peso e estatura e menor \%G em comparação às meninas $(p<0,05)$. Referente às variáveis motoras de impulsão horizontal, agilidade e abdominal os escores do sexo masculino são significativamente melhores do que o sexo feminino, em contrapartida, meninas apresentaram maiores valores de flexões de braço comparado aos meninos $(p<0,05)$. 
Tabela 1. Diferença entre os gêneros para as características antropométricas e do desempenho motor.

\begin{tabular}{lcc}
\hline \multicolumn{1}{c}{ Sexo } & $\begin{array}{c}\text { Meninos } \\
(\mathbf{n}=\mathbf{1 7})\end{array}$ & $\begin{array}{c}\text { Meninas } \\
(\mathbf{n}=\mathbf{3 0})\end{array}$ \\
\hline Idade (anos) & $14,2 \pm 1,4$ & $13,5 \pm 1,6$ \\
Massa Corporal $(\mathrm{Kg})$ & $64,0 \pm 10,7^{*}$ & $50,8 \pm 7,9$ \\
Estatura (m) & $1,73 \pm 0,06^{*}$ & $1,58 \pm 0,05$ \\
IMC (kg/m $\left.{ }^{2}\right)$ & $21,0 \pm 2,4$ & $20,1 \pm 2,6$ \\
Percentual de gordura (\%) & $21,1 \pm 5,9^{*}$ & $27,3 \pm 6,4$ \\
Impulsão horizontal (cm) & $185,1 \pm 22,0^{*}$ & $157,4 \pm 17,9$ \\
Flexão de braço (rep) & $14,7 \pm 7,7^{*}$ & $19,9 \pm 7,8$ \\
Flexibilidade & $24,5 \pm 11,1$ & $26,6 \pm 7,9$ \\
Agilidade (s) & $10,3 \pm 0,8^{*}$ & $11,4 \pm 0,5$ \\
Abdominal (rep) & $39,7 \pm 7,0^{*}$ & $31,4 \pm 5,4$ \\
\hline${ }^{*}$ Diferença significativa entre os sexos $(\mathrm{p}<0,05)$. & & \\
IMC - İndice de Massa Corporal & &
\end{tabular}

Considerando o estágio maturacional, não foi encontrada diferença significativa entre meninos púberes e pós-púberes em relação às variáveis antropométricas e do desempenho motor. No entanto, entre as meninas foram encontradas diferenças significativas $(p<0,05)$ na comparação de púberes e pós-púberes nas variáveis antropométricas, com exceção da estatura (Tabela 2).

Tabela 2. Freqüência de indivíduos púberes e pós-púberes e as médias e desvios padrão das características antropométricas e das variáveis do desempenho motor, entre os diferentes estados maturacionais.

\begin{tabular}{lcccc}
\hline & \multicolumn{2}{c}{ Meninos } & \multicolumn{2}{c}{ Meninas } \\
& Púbere & Pós-púbere & Púbere & Pós-púbere \\
& $47,1 \%$ & $52,9 \%$ & $86,7 \%$ & $13,3 \%$ \\
\hline Idade & $14,0 \pm 1,1$ & $14,4 \pm 1,7$ & $13,2 \pm 1,5^{*}$ & $15,0 \pm 1,4$ \\
MC $(\mathrm{Kg})$ & $63,3 \pm 11,8$ & $64,6 \pm 7,9$ & $49,1 \pm 6,2^{*}$ & $61,7 \pm 10,2$ \\
Estatura $(\mathrm{m})$ & $1,71 \pm 0,06$ & $1,75 \pm 0,06$ & $1,58 \pm 0,05$ & $1,59 \pm 0,04$ \\
IMC $\left(\mathrm{kg} / \mathrm{m}^{2}\right)$ & $21,3 \pm 3,3$ & $20,8 \pm 1,4$ & $19,5 \pm 1,9^{*}$ & $24,1 \pm 3,5$ \\
$\% \mathrm{GG}$ & $19,9 \pm 5,0$ & $22,1 \pm 6,8$ & $26,3 \pm 5,9^{*}$ & $34,3 \pm 5,4$ \\
IH $(\mathrm{cm})$ & $189,0 \pm 27,8$ & $181,7 \pm 16,2$ & $156,7 \pm 19,1$ & $161,7 \pm 6,3$ \\
FB $(\mathrm{rep})$ & $16,5 \pm 9,2$ & $13,1 \pm 6,3$ & $19,0 \pm 8,0$ & $25,5 \pm 1,7$ \\
Flexibilidade & $23,7 \pm 10,5$ & $25,3 \pm 12,1$ & $25,9 \pm 8,2$ & $31,5 \pm 4,5$ \\
Agilidade $(\mathrm{s})$ & $10,4 \pm 0,7$ & $10,2 \pm 0,9$ & $11,3 \pm 0,5$ & $11,5 \pm 0,2$ \\
Abdominal (rep) & $39,1 \pm 7,7$ & $40,2 \pm 6,7$ & $31,6 \pm 5,6$ & $30,5 \pm 3,6$ \\
\hline * Diferença significativa entre púberes e pós púberes (p<0,05) & & \\
MC - massa corporal; \% $\%$ - percentual de gordura; IMC - Índice de Massa Corporal; IH - impulsão \\
horizontal; FB - flexão de braço.
\end{tabular}

Considerando a amostra total, a Tabela 3 apresenta os valores de correlação entre as variáveis. Observou-se uma relação inversa $(p<0,05)$ entre o \%G e impulsão horizontal, além disso, a variável motora agilidade obteve relação positiva significativa com $\circ \% \mathrm{G}$, necessitando destacar que um menor escore para esta variável é indicador de melhor desempenho.

A correlação da maturação com a impulsão horizontal foi positiva $(p<0,05)$, indicando que indivíduos em maior estágio maturacional apresentam melhor desempenho para a variável motora de impulsão horizontal (Tabela 3).

$\mathrm{Na}$ Tabela 4, estão expressos os resultados da análise de regressão linear, tendo como variáveis dependentes a impulsão horizontal e agilidade. Em relação à impulsão horizontal, o sexo explicou $32,8 \%$ da variabilidade, e em conjunto com o \%G e a maturação, $36,5 \%$. Para a variável motora agilidade, a variabilidade foi explicada em $39,4 \%$ pelo sexo, e com o acréscimo do $\% \mathrm{G}$ e da maturação o percentual aumentou para $55 \%$. 
Tabela 3. Correlação entre o percentual de gordura, estágio maturacional e as variáveis do desempenho motor.

\begin{tabular}{ccccccc}
\hline & Maturação & IH & FB & Flex & Ag & Abdm \\
\hline \%G & 0,14 & $-0,42^{*}$ & 0,04 & $-0,006$ & $0,61^{*}$ & $-0,14$ \\
Maturação & & $0,32^{*}$ & $-0,17$ & 0,17 & $-0,27$ & 0,15 \\
IH & & & $0,31^{*}$ & $0,30^{*}$ & $-0,69^{*}$ & 0,18 \\
FB & & & & $0,39^{*}$ & $-0,08$ & $-0,08$ \\
Flex & & & & & $-0,26$ & $-0,16$ \\
Ag & & & & & & $-0,32^{*}$ \\
\hline
\end{tabular}

\%G - percentual de gordura; IH - impulsão horizontal; FB - flexão de braços; Flex - flexibilidade; Ag agilidade; Abdm - abdominal; * $p<0,05$.

Tabela 4. Modelos de regressão linear para a relação entre o sexo e as variáveis de impulsão horizontal e agilidade, ajustado pelo percentual de gordura e a maturação.

\begin{tabular}{|c|c|c|c|c|c|}
\hline $\begin{array}{c}\text { Variável } \\
\text { dependente }\end{array}$ & & $r$ & $\mathbf{R}^{2}$ & EPE & $\begin{array}{c}\text { Variáveis } \\
\text { independentes }\end{array}$ \\
\hline \multirow{3}{*}{$\begin{array}{l}\text { Impulsão } \\
\text { horizontal }\end{array}$} & Modelo 1 & 0,573 & 0,328 & 19,49 & $\begin{array}{l}\text { Intercepto } \\
\text { Sexo }\end{array}$ \\
\hline & Modelo 2 & 0,603 & 0,364 & 19,18 & $\begin{array}{c}\text { Intercepto } \\
\text { Sexo } \\
\% G\end{array}$ \\
\hline & Modelo 3 & 0,604 & 0,365 & 19,38 & $\begin{array}{c}\text { Intercepto } \\
\text { Sexo } \\
\% G \\
\text { Maturação }\end{array}$ \\
\hline \multirow{3}{*}{ Agilidade } & Modelo 1 & 0,628 & 0,394 & 0,65 & $\begin{array}{l}\text { Intercepto } \\
\text { Sexo }\end{array}$ \\
\hline & Modelo 2 & 0,732 & 0,536 & 0,58 & $\begin{array}{c}\text { Intercepto } \\
\text { Sexo } \\
\% G \\
\end{array}$ \\
\hline & Modelo 3 & 0,741 & 0,550 & 0,58 & $\begin{array}{l}\text { Intercepto } \\
\text { Sexo } \\
\% G \\
\text { Maturação }\end{array}$ \\
\hline
\end{tabular}

$\% G$ - percentual de gordura.

\section{Discussão}

Para as variáveis antropométricas os meninos apresentaram maior massa corporal e estatura, comparados às meninas, corroborando com outros estudos realizados com adolescentes (GUEDES; GUEDES, 1993; SALVADOR, 2005).

Observa-se que a grande maioria dos praticantes de handebol do sexo masculino, assim como do sexo feminino apresentaram escores classificados como moderadamente altos para valores absolutos do percentual de gordura (LOHMAN, 1992), com percentual médio de $21,1 \pm 5,9 \%$ e $27,3 \pm 6,4 \%$, respectivamente. Além disso, foi apontado menor $\% \mathrm{G}$ nos rapazes em relação às moças, resultado este também encontrado em atletas amadores de handebol adultos (GRANADOS et al., 2007; GOROSTIAGA et al., 2005) e em adolescentes sem nenhum tipo de prática esportiva (FARIAS, SALVADOR, 2005).

Assim, meninas ao fim da puberdade apresentam proporcionalmente $\mathrm{o}$ dobro de gordura em relação aos meninos (FARIAS et al., 2009), isto se deve possivelmente pelo fato de meninas apresentarem significativamente maior tamanho e número de células adiposas em relação aos meninos (CHUMLEA et al., 1981) e por sofrerem ação do hormônio estrogênio (VITOR et al., 2008).

Referente ao desempenho motor, o handebol é um esporte composto de movimentos que utilizam a combinação de habilidades motoras básicas do ser humano como correr, saltar e arremessar, deste modo, o treinamento destas variáveis torna-se imprescindível. No presente estudo, meninos apresentaram escores nos testes de impulsão horizontal, agilidade e 
abdominal significativamente melhor comparado às meninas, estando de acordo com resultados encontrados em outros estudos realizados com adolescentes (GUEDES; GUEDES, 1993; ANDERSEN et al., 2009).

O aumento da força muscular esta associada a hormônios anabólicos, em destaque os hormônios do crescimento e a testosterona, portanto, na adolescência por volta de 14 anos de idade, meninos apresentam evolução em algumas capacidades motoras, enquanto as meninas tendem a manter ou diminuir seus escores motores. Os meninos ganham força por influência dos hormônios masculinos, aumentando a massa muscular e diminuindo o \%G, e as meninas como citado anteriormente sofrem a ação de hormônios como o estrogênio, aumentando a gordura corporal conseqüentemente diminuindo $\mathrm{O}$ desempenho em testes motores (VITOR et al., 2008).

Entretanto, as meninas apresentaram em média melhores escores no teste de flexão de braços $(19,9 \pm 7,8$ meninas vs $14,7 \pm 7,7$ meninos), resultado este que não corrobora com outras evidências científicas (ANDERSEN et al., 2009), em contrapartida, no estudo de Silva (2002), indivíduos do sexo feminino com idades entre $8 \mathrm{e}$ 13 anos demonstraram superioridade nos valores de força de membros superiores em relação ao sexo masculino. Contudo, o modo em que o teste é realizado, pode facilitar o desempenho das meninas, assim como o incremento de força estimulado pela menarca, além disso, o controle da massa corporal e de componentes coordenativos nos testes de natureza motora é de fundamental importância, pois estas variáveis influenciam de forma significativa nos testes que objetivam avaliar o desempenho motor (RÉ et al., 2005).

Sobre as comparações entre sujeitos púberes e pós púberes, apenas entre as meninas púberes e pós-púberes foram encontradas diferenças significativas nas variáveis de peso, IMC e \%G. Segundo Ré et al. (2005), indivíduos em estágios maturacionais mais avançados apresentam maior massa corporal comparados com os mais tardios, estando de acordo com o presente estudo, além disso, tendem a ser mais altos. Portanto, pode-se explicar o maior valor médio do IMC $\left(\mathrm{Kg} / \mathrm{m}^{2}\right)$ em indivíduos pós-púberes.

Apesar do IMC não ser capaz de fornecer informações sobre a composição corporal e a distribuição de gordura total (GARN et al., 1986), foi observado que meninas em estágio maturacional avançado apresentam também maiores valores de \%G, em relação aos tardios. Estes resultados vão de encontro com outros estudos realizados com adolescentes (BAXTERJONES et al., 2008; BINI et al., 2000).

A literatura manifesta-se apontando que indivíduos em estágio maturacional avançado apresentam melhores desempenhos em testes motores comparado aos tardios (MALINA et al., 2000; HELSEN et al., 2000). Entretanto, não foi encontrada nenhuma diferença significativa entre os participantes púberes e pós-púberes para as variáveis do desempenho motor em ambos os sexos, porém, os indivíduos do estudo encontram-se entre os estágios de pilosidade pubiana II e V. Deste modo, talvez pudessem ser encontradas diferenças nas variáveis motoras entre os estágios maturacionais com a existência de participantes pré-púberes (estágio I).

Analisando os coeficientes de correlação simples, observa-se que apenas as variáveis motoras de impulsão horizontal $(r=-0,42)$ e agilidade $(r=0,61)$ apresentaram correlação significativa com o \%G, corroborando com os achados de Keogh et al. (2003) que observaram uma relação inversa entre $o$ percentual de gordura e os testes motores de velocidade de deslocamento e agilidade de jovens atletas de hockey. Nossos resultados também estão de acordo com estudo realizado por Ferreira $e$ Bohme (1998), os quais demonstraram que meninos e meninas apresentaram correlação inversa significativa entre $0 \% \mathrm{G}$ e 0 teste de impulsão horizontal.

Assim como o \%G, a maturação sexual apresentou correlação significativa apenas para as variáveis de impulsão horizontal e agilidade com valores de $r=0,31$ e $r=-0,30$, respectivamente. No estudo de Vitor et al. (2008) realizado com jovens atletas do sexo masculino, foram encontrados aumentos nos resultados de força de membros inferiores (Impulsão horizontal) juntamente com o avanço de cada estágio maturacional, enquanto que para a agilidade os resultados foram contrários, considerando que o menor valor deste teste indica melhor desempenho.

A variabilidade da impulsão horizontal foi explicada pelo sexo em $32,8 \%$, e em conjunto com o \%G e a maturação biológica, o efeito 
preditor aumentou para $36,5 \%$. Neste sentido, leva-se a especulação de que o sexo do indivíduo, junto com $\mathrm{o}$ processo de desenvolvimento maturacional e baixos valores do $\%$, favorece o desempenho no teste de impulsão horizontal. Em um estudo realizado em freqüentadores de um programa de iniciação esportiva, o componente motor de força de membros inferiores apresentou variabilidade de $53 \%$ explicada pela idade cronológica juntamente com a estatura (RÉ et al., 2005). E ainda, o pico de velocidade de crescimento em conjunto com os estágios de pilosidade pubiana explicou a variabilidade do teste de impulsão horizontal em $47 \%$, para praticantes de futebol (MACHADO et al., 2009).

O componente motor de agilidade, no modelo de regressão apresentou maior coeficiente de determinação, pelo sexo $(39,4 \%)$ e em conjunto com o \%G e a maturação (55\%). Apesar destes valores, sugere-se que estudos longitudinais devem ser realizados, para afirmar com precisão o efeito preditor da agilidade, pois o componente coordenativo pode influenciar no resultado (RÉ et al., 2005), além disso, indivíduos na faixa etária estudada não têm dependência elevada do processo de crescimento e desenvolvimento como teriam crianças de faixa etárias menores.

Limitações podem ser apontadas no presente estudo, como a quantidade de massa muscular que não foi controlada, onde é evidenciado o aumento desta variável conforme 0 desenvolvimento maturacional (BAXTER-JONES et al., 2008), podendo ainda dificultar interpretações dos efeitos nos testes motores. E ainda, a experiência de prática do esporte específico também pode ser um fator de influência nas variáveis motoras analisadas. A escassez de estudos que buscam analisar adolescentes praticantes de handebol é ampla, principalmente no Brasil. Portanto, sugere-se que estudos futuros sejam realizados com o objetivo de investigar as relações do desempenho motor com a maturação biológica, composição corporal, experiência de prática, além de outros aspectos que devem ser considerados para estudos com esta população.

Em conclusão, os meninos são mais altos e mais pesados comparados as meninas, porém, apesar de apresentar maior peso, os valores do $\% G$ são mais baixos. Referente às diferenças no desempenho motor entre os gêneros, os rapazes apresentaram melhores escores para os testes motores de impulsão horizontal, agilidade e abdominal em relação às moças, porém, no teste de flexão de braços meninas apresentaram valores mais elevados.

Embora tenham sido encontrados resultados significativos, a contribuição preditora do sexo isolado e juntamente com o percentual de gordura $(\% \mathrm{G})$ e a maturação biológica apresentou baixos valores. Neste sentido, as variações ocorridas na impulsão horizontal e agilidade, podem não ser atribuídas apenas às variáveis independentes analisadas.

\section{Referências}

ALTINI NETO A.; PELLEGRINOTTI I. L.; MONTEBELO M. I. L. Efeitos de um programa de treinamento neuromuscular sobre o consumo máximo de oxigênio e salto vertical em atletas iniciantes de voleibol. Revista Brasileira de Medicina do Esporte. v.12, n.1, p.33-38, 2006. Disponível em: http://www.scielo.br/pdf/rbme/v12n1/v12n1a07.pdf . Acesso em: 23 de out. 2009.

ANDERSEN L. B.; LAWLOR D. A.; COOPER A. R.; FROBERG K.; ANDERSSEN S. A. Physical fitness in relation to transport to school in adolescents: the Danish youth and sports study. Scandinavian Journal of Medicine Science Sports. v.19, p.406-411, 2009.

BAXTER-JONES A. D. G.; EISENMANN J. C.; MIRWALD R. L.; FAULKNER R. A.; BAILEY D. A. The influence of physical activity on lean mass accrual during adolescence: a longitudinal analysis. Journal of Applied Physiology. v.105, p.734-741, 2008.

BINI V.; CELI F.; BERIOLI M. G.; BACOSI M. L.; STELLA P.; GIGLIO P.; TOSTI L.; FALORNI A. Body mass index in children and adolescents according to age and pubertal stage. European Journal of Clinical Nutrition. v.54, p.214-218, 2000.

CHUMLEA W. C.; KNITTLE J. L.; ROCHE A. F.; SIERVOGEL R. M.; WEBB P. Size and number of adipocytes and measures of body fat in boys and girls 10 to 18 years of age. American Journal of Clinical Nutrition. p.1791-1797, 1981.

FARIAS E. S.; SALVADOR M. R. D. Antropometria, composição corporal e atividade física de escolares. Revista Brasileira de Cineantropometria e Desempenho Humano. v.7, n.1, p.21-29, 2005. Disponível em: http://www.rbcdh.ufsc.br/MostraEdicao.do?edicao $\underline{=17}$. Acesso em: 15 ago. 2009. 
FARIAS E. S.; PAULA F.; CARVALHO W. R. G.; GONÇALVES E. M.; BALDIN A. D.; GUERRAJUNIOR G. Efeito da atividade física programada sobre a composição corporal em escolares adolescentes. Jornal de Pediatria. v.85, n.1, p.28-34, 2009. Disponível em:

http://www.scielo.br/scielo.php?script=sci issuetoc \&pid=0021755720090001\&lng=pt\&nrm=iso. Acesso em: 08 out. 2009.

FERREIRA M.; FRANÇA N. M.; SOUZA M. T.; MATSUDO V. K. R. Comparação da aptidão física de escolares de Itaquera (zona leste - São Paulo) e São Caetano do Sul. Revista Brasileira de Ciência e Movimento. v.4, n.2, p.19-27, 1990. Disponível em:

http://portalrevistas.ucb.br/index.php/RBCM/issue/ view/19. Acesso em: 20 jun. 2009.

FERREIRA M, BÖHME MTS. Diferenças sexuais no desempenho motor de crianças: Influência da adiposidade corporal. Revista Paulista de Educação Física. v.12, n.2, p.181-192, 1998. Disponível em:

http://www.usp.br/eef/rpef/v12n2/v12n2.htm. Acesso em: 13 fev. 2009.

GARN S. M.; LEONARD W. R.; HAWTHORNE V. $M$. Three limitations of the body mass index. American Journal of Clinical Nutrition. v.44, p.996-997, 1986.

GORDON C. C.; CHUMLEA W. C.; ROCHE A. F. Stature, Recumbent, Length, and Weigth. In: LOHMAN T. G.; ROCHE A. F.; MARTORELL R. Anthropometric standardization reference manual. Champaign: Human Kinetics; 1991. p. 38.

GOROSTIAGA E. M.; GRANADOS C.; IBANEZ J.; IZQUIERDO M. Differences in physical fitness and throwing velocity among elite and amateur male handball players. International Journal of Sports Medicine. v.26, p.225-232, 2005.

GUEDES D. P.; GUEDES J. E. R. P. Manual prático para avaliação em Educação Física. São Paulo: Manole; 2006.

GUEDES D. P.; GUEDES J. E. R. P. Crescimento e desempenho motor em escolares do município de Londrina, Paraná, Brasil. Cadernos de Saúde Pública. v.9, supl. 1, p.58-70, 1993. Disponível em:

http://www.scielo.br/scielo.php?script=sci issuetoc \&pid=0102311X19930005\&lng=pt\&nrm=iso. Acesso em: 17 jan. 2009.

GRANADOS C.; IZQUIERDO M.; IBANEZ J.; BONNABAU H.; GOROSTIAGA E. M. Differences in physical fitness and throwing velocity among elite and amateur female handball players.
International Journal of Sports Medicine. v.28, p.860-867, 2007.

HELSEN W. F.; HODGES N. J.; VAN WINCKEL J.; STARKES J. L. The roles of talent, physical precocity and practice in the development of soccer expertise. Journal of Sports Science. v.18, p.727-736, 2000.

KEOGH J. W. L.; WEBER C. L.; DALTON C. T. Evaluation of anthropometric, physiological, and skill-related tests for talent identification in female field hockey. Canadian Journal of Applied Physiology. 2003; v.28, n.3, p.397-409.

KRAEMER W. J.; VOLER J. S.; CLARK K. L.; GORDON S. E.; PUHL S. M.; KOZIRIS L. P.; MCBRIDE J. M.; TRIPLETT-MCBRIDE N. T.; PUTUKIAN M.; NEWTON R. U.; HAKKINEN K.; BUSH J. A.; SEBASTIANELLI W. J. Influence of exercise training on physiological and performance changes with weight loss in men. Medicine and Science in Sports Exercise. v.31, n.9, p.1320-1329, 1999.

LOHMAN T. G. Advances in body composition assessment. Champaign(II): Human Kinetics; 1992.

MACHADO D. R. L.; BARBANTI V. J. Maturação esquelética e crescimento em crianças e adolescentes. Revista Brasileira de Cineantropometria e Desempenho Humano. v.9, n.1, p.12-20, 2007. Disponível em: http://www.rbcdh.ufsc.br/MostraEdicao.do?edicao =28. Acesso em: 24 mai. 2009.

MACHADO D. R. L.; BONFIM M. R.; COSTA L. T. Pico de velocidade de crescimento como alternativa para a classificação maturacional associada ao desempenho motor. Revista Brasileira de Cineantropometria e Desempenho Humano. v.11, n.1, p.14-21, 2009. Disponível em: http://www.rbcdh.ufsc.br/MostraEdicao.do?edicao =39. Acesso em: 25 jul. 2009.

MALINA RM, BOUCHARD C. Growth, Maturation, and Physical Activity. Champaign (II): Human Kinetics; 1991.

MALINA R. M.; PEÑA REYES M. E.; EISENMANN J. C.; HORTA L.; RODRIGUES J.; MILLER R. Height, mass and skeletal maturity of elite Portuguese soccer players aged 11-16 years. Journal of Sports Science. v.18, p.685-693, 2000.

MALINA R. M.; DOMPIER T. P.; POWELL J. W.; BARRON M. J.; MOORE M. T. Validation of a noninvasive maturity estimate relative to skeletal age in youth football players. Clinical Journal of Sports Medicine. v.17, n.5, p.362-368, 2007. 
MARTIN R. H. C.; UEZU R.; PARRA A. S.;

ARENA S. S.; BOJIKIAN L. P.; BÖHME M. T. S. Auto-avaliação da maturação sexual masculina por meio da utilização de desenhos e fotos.

Revista Paulista de Educação Física. v.15, n.2, p.212-222, 2001. Disponível em:

http://www.usp.br/eef/rpef/Sumar152.htm. Acesso em: 12 jan. 2009.

RÉ A. H. N.; BOJIKIAN L. P.; TEIXEIRA C. P.; BÖHME M. T. S. Relações entre crescimento, desempenho motor, maturação biológica e idade cronológica em jovens do sexo masculino.

Revista Brasileira de Educação Física e Esporte. v.19, n.2, p.153-162, 2005. Disponível em:

http://www.usp.br/eef/rbefe/v19n22005/sumariov1 9n2.htm. Acesso em 10 jan. 2009.

SILVA R. J. S. Características de crescimento, composição corporal e desempenho físico relacionado a saúde em crianças $e$ adolescentes de 07 a 14 anos da região do Cotinguiba (SE). 2002. 114 f. Dissertação (Mestrado em Educação Física) - Universidade Federal de Santa Catarina, Florianópolis, 2002.

SLAUGHTER M. H.; LOHMAN T. G.; BOILEAN C. A.; STILLMAN R. J.; VANVOAN M. E.; BEMEBN D. A. Skinfold equations for estimation of body fatness in children and youth. Human Biology. v.60, n.5, p.709-723, 1988.

TANNER JM. Growth at adolescence. $2^{\mathrm{a}}$ ed Oxford: Blackwell Scientific; 1962.

VITOR F. M.; UEZU R.; SILVA F. B. S.; BÖHME M. T. S. Aptidão física de jovens atletas do sexo masculino em relação à idade cronológica e estágio de maturação sexual. Revista Brasileira de Educação Física e Esporte. v.22, n.2, p.139148, 2008. Disponível em: http://www.usp.br/eef/rbefe/v22n22008/sumariov2 2n2psite.htm. Acesso em: 09 mai. 2009.

Comitê de ética da Universidade Federal do Paraná: 3185.0.000.091-08

\section{Endereço:}

Rodolfo André Dellagrana

Departamento de Educação Física

Rua Coração de Maria, 92, Jardim Botânico

Curitiba Paraná Brasil

80215-370

e-mail: radellagrana@yahoo.com.br

Recebido em: 11 de dezembro de 2009.

Aceito em: 14 de junho de 2010.

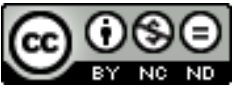

Motriz. Revista de Educação Física. UNESP, Rio Claro, SP, Brasil - elSSN: 1980-6574 - está licenciada sob Licenca Creative Commons 